\section{End of the line for cannabinoid receptor 1 as an anti-obesity target? An opinion}

\section{Maurizio Bifulco and Simona Pisanti}

The recent News and Analysis article, "End of the line for cannabinoid receptor 1 as an antiobesity target?" (Nature Rev. Drug Discov. 7, 961-963; 2008) $)^{1}$ highlighted the downfall of cannabinoid receptor 1 (CB1) blockers for the treatment of obesity, largely owing to psychiatric side effects. In this correspondence, we consider whether it seems possible to save the CB1 blocking strategy, and the steps that might be needed to achieve this.

First, CB1 blockers that do not cross the blood-brain barrier need to be developed. Their application to pathologies in which blockade of $\mathrm{CB} 1$ receptors in the central nervous system is required would therefore be excluded, limiting the anti-obesity efficacy to an improvement of metabolic factors that can lead to diabetes and cardiovascular diseases, independently of central control. In this respect, it is noteworthy that peripheral blockade of $\mathrm{CB} 1$ receptors has been reported to have a therapeutic effect in several pathologies, such as hepatic fibrosis, chronic inflammatory conditions, diabetes and cancer ${ }^{2,3}$. So, in these diseases, highly selective peripheral $\mathrm{CB} 1$ antagonists, the use of lower doses or local administration of CB1 blockers could be strategies to minimize psychotropic side effects.

The potential of targeting the CB1 receptor in cancer therapy is particularly intriguing. CB1 blockers might be used in combination with chemotherapy, in order to reduce doses, avoid resistance and exert a more potent clinical effect. Interestingly, obesity is a well-recognized factor associated with both increased cancer incidence and unfavourable prognosis in several cancers ${ }^{4}$. Obesity, the weight gain following chemotherapy and the frequency of cancer recurrence linked to this weight gain make strategies to mitigate the adverse metabolic effects of obesity a priority for cancer prevention and treatment. So, CB1 blockers, which possess anti-obesity, anti-inflammatory and anticancer properties, might be a promising treatment option. Moreover, it would be interesting to perform observational studies on patients treated with $\mathrm{CB} 1$ blockers for a substantial period of time, to determine whether there is an association between the therapy and the incidence of all and site-specific cancers, which might indicate a protective effect of CB1 blockade.

Further studies are needed to assess the potential of CB1 blockers as anticancer drugs, as well as their possible application in other peripheral pathologies. For example, recent studies have provided evidence of a role for $\mathrm{CB} 1$ receptor signalling in angiogen$\mathrm{esis}^{5}$. Because angiogenesis is a fundamental process in cancer progression and in the pathogenesis of ocular, joint and skin disorders, for which a locally delivered therapy is possible, redirecting research efforts towards the effects of CB1 blockers on such disorders merits consideration.

Finally, the incidence of depression resulting from treatment with the $\mathrm{CB} 1$ blocker rimonabant (Acomplia; SanofiAventis) is more pronounced in patients with a previous history of depression. Variations in the coding or promoter regions of the gene encoding CB1 (CNR1) have been associated with mood disorders and predisposition to depression ${ }^{6}$. Our hypothesis is that inter-individual differences in the efficacy and psychiatric side effects of CB1 blockers could be related to these variations, and so selecting patients on the basis of $\mathrm{CB} 1$ receptor expression or $C N R 1$ variants might reduce the risk of adverse side effects.

Overall, in our opinion, such potential avenues for the future application of $\mathrm{CB} 1$ receptor modulators indicate that the endocannabinoid system and its regulation remains a promising target for pharmacotherapy.

Maurizio Bifulco and Simona Pisanti are at the Department of Pharmaceutical Sciences, University of Salerno, Italy. Correspondence to M.B. e-mail:maubiful@unisa.it

1. Jones, D. End of the line for cannabinoid receptor 1 as an anti-obesity target? Nature Rev. Drug Discov. 7 961-963 (2008)

2. Bifulco, M., Grimaldi, C., Gazzerro, P., Pisanti, S. \& Santoro, A. Rimonabant: just an antiobesity drug? Current evidence on its pleiotropic effects. Mol. Pharmacol. 71, 1445-1456 (2007)

3. Bifulco, M., Malfitano, A. M., Pisanti, S. \& Laezza, C. Endocannabinoids in endocrine and related tumours. Endocr. Relat. Cancer 15, 391-408 (2008).

4. Bianchini, F., Kaaks, R. \& Vainio H. Overweight, obesity, and cancer risk. Lancet Oncol. 3, 565-574 (2002).

5. Pisanti, S. et al. Antiangiogenic activity of the endocannabinoid anandamide: correlation to its tumor-suppressor efficacy. J. Cell Physiol. 211, 495-503 (2007).

6. Barrero, F. J. et al. Depression in Parkinson's disease is related to a genetic polymorphism of the cannabinoid receptor gene (CNR1). Pharmacogenomics J. 5, 135-141 (2005). 